\title{
PLANETARY CAMERA OBSERVATIONS OF NGC 1275: DISCOVERY OF A CENTRAL POPULATION OF COMPACT MASSIVE BLUE STAR CLUSTERS ${ }^{1}$
}

Jon A. HoltZMAN

Lowell Observatory, 1400 Mars Hill Road, Flagstaff, Arizona 86001

\section{S. M. FABER}

UCO/Lick Observatories, Board of Studies in Astronomy and Astrophysics, University of California, Santa Cruz, California 95064

EDWARD J. SHAYA

Department of Physics and Astronomy, University of Maryland, College Park, Maryland 20742

TOD R. LAUER

National Optical Astronomy Observatories, ${ }^{2}$ P.O. Box 26732, Tucson, Arizona 85726

EDWARD J. GRoth

Department of Physics, Princeton University, Box 708, Princeton, New Jersey 08544

DEIDRE A. HunTER

Lowell Observatory, 1400 Mars Hill Road, Flagstaff, Arizona 86001

William A. BAUM

Department of Astronomy, FM-20, University of Washington, Seattle, Washington 98195

$$
\text { S. P. Ewald }
$$

Space Telescope Science Institute, 3700 San Martin Drive, Baltimore, Maryland 21218

\section{J. JEFF HESTER}

Department of Physics and Astronomy, Arizona State University, Tempe, Arizona 85287

RoBERT M. LIGHT

UCO/Lick Observatories, Board of Studies in Astronomy and Astrophysics, University of California, Santa Cruz, California 95064

C. ROGER LYNDS AND EARL J. O'NeIL, JR.

National Optical Astronomy Observatories, ${ }^{2}$ P. O. Box 26732, Tucson, Arizona 85726

JAMES A. WESTPHAL

Division of Geological and Planetary Sciences, 170-25, California Institute of Technology, Pasadena, California 91125 Received 14 October 1991

\begin{abstract}
We have discovered a population of bright blue pointlike sources within $5 \mathrm{kpc}$ of the nucleus of NGC 1275 using $H S T$ Planetary Camera observations. The typical object has $M_{V} \sim-12$ to $-14\left(H_{0}=75\right.$ $\mathrm{km} \mathrm{s}^{-1} \mathrm{Mpc}^{-1}$ ); the brightest has $M_{V} \sim-16$. They are all blue, with $V-R \leqslant 0.3$. The color distribution and lack of excess $\mathrm{H} \alpha$ emission are consistent with nearly all being continuum sources. Many of the sources are unresolved even with the $H S T$ and consequently have sizes of $\lesssim 15 \mathrm{pc}$. We suggest that these are young star clusters that will evolve to look like globular clusters. They are bluer than any clusters seen in the Milky Way or M87, and brighter than the blue clusters seen in the LMC. We derive ages of several hundred million years or less and corresponding masses of $10^{5}-10^{8} \mathscr{M} \odot$. The existence of these young clusters may be connected with a current or previous interaction with another galaxy, with the cooling flow in NGC 1275, or with some combination. Structure is detected in the underlying galaxy light that is suggestive of a merge between NGC 1275 and a second galaxy some $10^{8} \mathrm{yr}$ ago. If this merger triggered star formation, it would naturally account for the observed uniformity of cluster colors. Steady-state star formation in the x-ray cooling flow would imply a wider range in cluster age and color than is seen, unless the clusters disrupt. An interaction with the projected high-velocity, infalling system cannot explain the observations because this system has not yet reached the center of NGC 1275 where the clusters are concentrated, and because it has a total interaction time that is far too short for either the observed cluster lifetimes or the dynamical lifetime of structure in the galaxy. If the presence of recently formed protoglobulars around NGC 1275 is related to a previous merger, this would remove an important objection to the merger hypothesis for elliptical galaxy origins, provided that adequate gas is available in the merger for their formation.
\end{abstract}

\footnotetext{
${ }^{1}$ Based on observations with the NASA/ESA Hubble Space Telescope, obtained at the Space Telescope Science Institute, which is operated by AURA, Inc., under NASA Contract No. NAS 5-26555.

${ }^{2}$ Kitt Peak National Observatory, National Optical Astronomy Observatories are operated by the Association of Universities for Research in Astronomy, Inc., under cooperative agreement with the National Science Foundation.
} 


\section{INTRODUCTION}

NGC 1275 (Perseus A) has been extensively studied in the last several decades and is known to be unusual in several respects. It is the central galaxy of the Perseus cluster (Abell 426) and the brightest galaxy within $15000 \mathrm{~km} / \mathrm{s}$ (Lauer \& Postman 1991). It has a pointlike nucleus with a Seyfert-like spectrum and is a strong radio source (Khachikian \& Weedman 1974). Although it is classified as an elliptical galaxy, much structure has been detected, particularly the presence of gaseous filaments extending to large radii (Lynds 1970) which emit in $\mathrm{H} \alpha$; the galaxy is also notably patchy with apparent dusty regions. Gas is present in the direction of the galaxy at two distinct velocities, $5200 \mathrm{~km} / \mathrm{s}$ (the velocity of the underlying galaxy) and $8200 \mathrm{~km} / \mathrm{s}$ (Minkowski 1957; Burbidge \& Burbidge 1965; Cowie et al. 1983). The highvelocity gas lies mostly to the north and northwest of the galaxy center. $\mathrm{H}_{\text {I }} 21 \mathrm{~cm}$ observations of the radio source show the high-velocity system in absorption, implying that this system lies in front of NGC 1275, despite its larger recession velocity (De Young et al. 1973); absorption by dust in this system also supports this. The Perseus cluster contains x-ray emitting gas centered around NGC 1275, and the observed brightness and temperature of this gas imply that a cooling flow may exist into the galaxy, with an estimated mass accretion rate of $\sim 200 \mathscr{M}_{\odot} / \mathrm{yr}$ (Fabian et al. 1981).

Because of the implied cooling flow, much thought has been given to possible fates for the infalling material. In particular, the possibility that the material forms stars has been proposed (e.g., McNamara \& O'Connell 1989). Several observations imply the presence of a young stellar population in NGC 1275. Hydrogen absorption has been seen in some, but not all, regions of the galaxy (Rubin et al. 1977; van den Bergh 1972). A composite spectrum of the entire central region implies that approximately $20 \%$ of the light at visible wavelengths is contributed by B-type stars (Wirth et al. 1983). A strong color gradient, in the sense that the galaxy is bluer toward the center, has been detected (Romanishin 1987); most cluster ellipticals have a gradient of the opposite sense. IUE observations of NGC 1275 show contributions from young stars (Nörgaard-Nielsen et al. 1990). The color gradient, IUE observations, and analysis of an $\mathrm{H}$ II-like region, discussed below, have all been used to infer star formation rates (SFRs). Almost all approaches have large uncertainties, but all imply star formation rates about an order of magnitude smaller than the mass accretion rates derived for the cooling flow. This has led to speculation that the IMF may be skewed toward more low-mass stars (since all the techniques for measuring SFRs are sensitive only to massive stars), or that energy input from the star formation influences the cooling flow, reducing the mass accretion rate.

A recent discovery is of particular relevance to the observations reported here. Shields \& Filippenko (1990; Shields et al. 1990) have detected an $\mathrm{H}$ II-like region at $18.5^{\prime \prime}$ from the nucleus of the galaxy at a position angle of $62 \mathrm{deg}$. After background subtraction, they find a very blue continuum source, which they believe is a young star cluster. For this object, they derive a mass of $5 \times 10^{6} \mathscr{M}_{\odot}$ assuming a standard initial mass function (IMF) and that the light is dominated by O-type stars.

Here we report on Planetary Camera observations of NGC 1275. The resolution of HST has enabled us to detect a population of bright blue point sources within 15 arcsec of the galaxy nucleus. We believe that these are massive young star clusters, all of a comparable age. Using population syn- thesis models, we derive ages of 300 million years or less. The colors and brightnesses are consistent with the prediction that these objects will evolve into typical Milky Way globular clusters, except that they may have higher metallicities. In addition to the point sources, there are about a dozen diffuse sources, of otherwise similar brightness and color. Most of our objects seem to differ from the Shields-Filippenko object, since it appears to have much stronger line emission.

We have also confirmed structure in the galaxy indicative of a possible merger event in NGC 1275 a few hundred million years ago. The rough equality of this timescale with the age limit on the clusters suggests that the cluster formation may have been triggered by this merger. The idea that NGC 1275 is a merger remnant adds to the list of known peculiarities of this object but offers some new ideas for its origin. These are discussed and compared in the final section.

\section{OBSERVATIONS}

Observations of NGC 1275 were obtained with the Planetary Camera on 20 February 1991 in four different filters: F555W, F702W, F664N, and F673N, with exposure times of $700,700,900$, and $900 \mathrm{~s}$. The first two are broadband filters centered in the green and red, respectively. The other filters are narrow and were chosen to cover the $\mathrm{H} \alpha$ line in the two velocity systems. For each filter, two identical exposures of the above exposure times were obtained to assist in removing cosmic rays. The broadband pictures are well exposed but are saturated in the galaxy core and in the core of a nearby star image. The narrowband observations have low signal to noise. The core of the galaxy lies near the center of the PC6 chip. Observations of a nearby star for use in understanding the PSF were attempted but failed because of $H S T$ pointing problems.

The images were processed using the techniques discussed by Lauer (1989) employing calibration data obtained near in time to the observations. An A-to-D correction was applied, and a bias level was subtracted using the information from the overscan region. Preflash, bias structure, and dark frames were subtracted, and the data were flattened with flatfields constructed from several observations of the bright Earth. Flats were available for the F555W and F702W filters. For the other filters, an average of these two flats was used to approximate the detector response at the intermediate wavelengths. We believe that the broadband flats are accurate to better than $5 \%$, while the narrowband flats may be somewhat poorer.

For each frame, cosmic rays were removed by comparing the pairs of exposures. This works well for extended sources if the frames are well registered, but has problems in the cores of point sources. For this reason, the frames were visually inspected beforehand, and all point sources were marked. Any point sources lying within a few pixels of a cosmic ray were flagged, and measurements on that source were subsequently ignored. After cosmic ray removal, the frames were added; for all four filters the registration between the pairs was sufficiently accurate that no shifting was required. There is, however, a shift between the observations with different filters.

\section{ANALYSIS}

\subsection{Brightnesses and Colors of Cluster Sources}

An $800 \times 800(35 \times 35$ arcsec $)$ section of the F555W exposure is shown in Fig. 1(a) [Plate 49] with logarithmic 
stretch; Fig. 1(b) [Plate 50] shows the central $18 \times 18$ arcsec region enlarged. For $H_{0}=75 \mathrm{~km} \mathrm{~s}^{-1} \mathrm{Mpc}^{-1}$ (adopted throughout), 1 arcsec corresponds to $0.34 \mathrm{kpc}$. The bright nucleus is the left of the two point sources; the right one is a foreground F star (Hughes \& Robson 1991). The prominent PSF structure in the nucleus demonstrates its pointlike nature. In addition, there are numerous pointlike sources surrounding the nucleus of NGC 1275 . These immediately caught our attention because they are significantly brighter than globular clusters in comparable observations of M87, despite the fact that NGC 1275 is more than 4 times more distant. These sources are more centrally concentrated than the galaxy light; almost all of them are found within 15 arc$\sec (5 \mathrm{kpc})$ of the nucleus, with about half of them within 10 arcsec.

On the F555W exposure, about 60 distinct objects were marked; most were found on PC6 (the chip containing the nucleus), but several were found on other chips. Automatic detection was avoided because of PSF structure in the wings of the nucleus and the bright star, remnant faint cosmic rays, and the presence of diffuse structure. Several slightly diffuse objects were also marked. It is likely that our visual marking is incomplete at faint limits. Coordinate shifts between the frames in the other colors were determined from the brightest pointlike objects, then the entire list from the F555W frame was transformed to the coordinates of the other frames. Almost all of the objects can be seen on the F702W frame, but only the brightest ones are visible on the narrowband frames.

Brightnesses were obtained using aperture photometry. To obtain background values, a second-order polynomial surface was fit to a 50 by 50 region centered on each object, excluding the area including and immediately surrounding the object. The objects were then recentroided and measured on this subtracted region. Profile fitting was not done because we do not have an accurate representation of the PSF, especially for the image cores, and because some of the objects are diffuse.

Since most of the objects are faint and the background is uncertain, we used small apertures, of radii 2 and 4 pixels, for the measurements. These gave comparable results, and we subsequently show only the 2 pixel measurements. Transformation to standard magnitudes requires an aperture correction to determine the total light in the objects, plus a calibration of the WFPC system to a ground-based system. The aperture correction is very difficult to obtain because stars have extended skirts from the $H S T$ spherical aberration and also because the PSF varies with location in the chip and with time (Holtzman et al. 1991). These effects create problems at about the $10 \%$ level for small apertures. However, much of the error comes from PSF variations, which cancels out for the colors because the observations were registered to within a few pixels.

For the NGC 1275 observations, aperture corrections were calculated in two ways. First, a moderately well exposed star near the edge of PC5 was measured. This gave $8.5 \%$ of the light within a 2 pixel radius in both $\mathrm{F} 555 \mathrm{~W}$ and F702W. In addition, a model PSF was constructed for the appropriate location of the nucleus of NGC 1275 in PC6, using obscuration information obtained from phase retrieval work on $H S T$ images. This model gave $7.4 \%$ and $7.8 \%$ in F555W and F702W. We adopted $8 \%(2.74 \mathrm{mag})$ as the aperture correction for both filters and believe that the corrections are uncertain at the 5\%-10\% level.
Observations of a ground-calibrated field in $\omega$ Cen were used for the transformation to ground-based magnitudes. These were available for F555W and F702W only. Accurate transformations are difficult to determine because of the problem with aperture corrections mentioned above, and also because many of the standard stars are underexposed. Because of focus changes, different aperture corrections were used for NGC 1275 and $\omega$ Cen. The scatter from the flight-to-ground transformation in the WF/PC filters is $5 \%-10 \%$. The uncertainties currently prevent us from determining a color term. The ground measurements of Harris et al. (1990) were used to determine the transformations between ground F555W and $V$ and ground F702W and $R$, with color terms a function of $V-R$; this transformation, using $30(21)$ stars, gave residual $1 \sigma$ errors per star of 0.02 $(0.022)$ magnitudes in F555W (F702W). Since the groundbased measurements are tied to Landolt standards, all of the $R$ measurements refer to Cousins $R$.

Because of the uncertainties in calibration, the magnitude zero point for each filter is subject to serious systematic errors: the uncertainty in aperture correction for the NGC 1275 frames and the uncertainty in the flight-to-ground photometric transformation. In a worst case, we estimate that the magnitudes and colors could be in error by as much as $0.15 \mathrm{mag}$; accurate zero points are extremely difficult to determine using HST! Note, however, that the internal measurements are affected only by normal errors such as photon statistics. This allows us to say that objects accurately appear to have the same color, without being able to determine precisely what that color is.

The color index zero point can be checked by determining the observed color of the nearby $F$ star or by comparing galaxy colors with those obtained from the ground. Unfortunately, the central few pixels of the $F$ star are saturated in both F555W and F702W, and the underlying galaxy contributes much light to that star, rendering accurate measurements of the star difficult. We estimated the color from the wings of the star and determined $(V-R) \sim 0.38$. This star has spectral type F4-F6 (Hughes \& Robson 1991), which should have $(V-R) \sim 0.27$. For the galaxy, the situation is complicated by the possible presence of a color gradient convolved with a drastically different PSF between the ground and $H S T$. We estimated its color using the ground-based $B$ and $R$ frames of Lauer \& Postman (1991), for a region of roughly constant color and free of point sources located several arcseconds to the SW of the nucleus. For this region, we obtain $(B-R) \approx 1.20$, compared to $(V-R) \approx 0.53$ from the $P C$ pictures. If we take the $B, V$, and $R$ colors to approximate those of normal stars (Bessell 1979), we find by interpolation that $(B-R)=1.20$ implies $(V-R)=0.43$. Consequently, both the F star and galaxy suggest that our measured $V-R$ colors may be too red by $0.1 \mathrm{mag}$.

The transformed $V$ and $R$ magnitudes are presented in Fig. 2 as a plot of color versus brightness. The error bars are from photon statistics in the object and background. The open boxes represent objects on the PC6 chip; objects on the other chips (i.e., further from the galaxy nucleus) are denoted by stellated points. Objects which appear diffuse to the eye are marked with circles, and the Shields and Filippenko object is shown with a filled circle. No correction has been applied for galactic or internal reddening within NGC 1275; consequently, the true colors may be significantly bluer than plotted. Even without a reddening correction and with zeropoint uncertainties, it is clear that there is a population of 


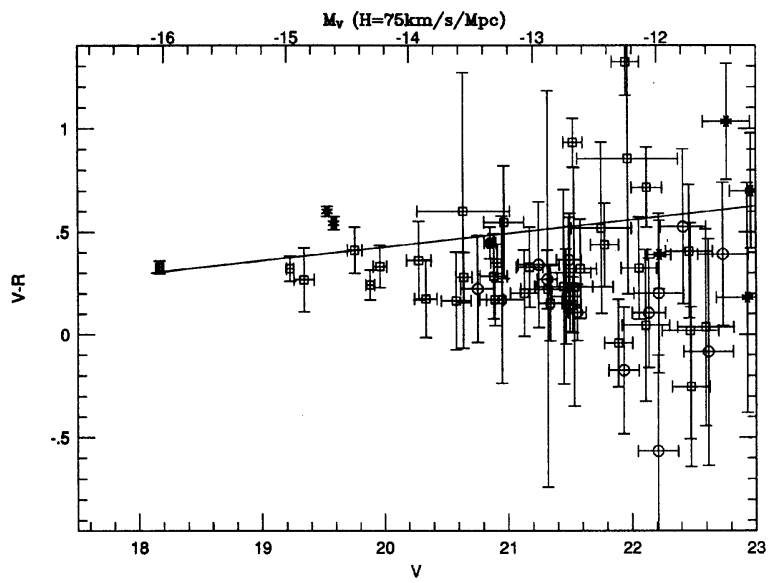

FIG. 2. $V-R$ color vs $V$ brightness of pointlike sources. Stellated points are objects falling on a chip other than PC6 (i.e., further from the nucleus). Visibly diffuse structures are shown with open circles. The Shields-Filippenko object is shown by a filled circle. The line shows an approximate fading and reddening vector from the population synthesis models in Fig. 7.

bright blue sources around NGC 1275. For a distance modulus of 34.2 , most of the objects have $M_{V} \sim-12$ to -14 , while the brightest object has $M_{V} \sim-16$; the absolute magnitudes are shown on the top scale of Fig. 2. Table 1 summarizes the object identifications, positions relative to the nuclear point source, magnitudes, and colors.

All objects seem to be about the same color, with $V-R \sim 0.3$. Burstein \& Heiles (1984) give a reddening estimate in the direction of NGC 1275 of $A_{V}=0.54 \mathrm{mag}$, which gives $\mathrm{E}(V-R)=0.13$ using the reddening curve of Cardelli et al. (1989); this value of the reddening is also supported by the analysis of Lynden-Bell et al. (1988). Consequently, the true colors of our objects are at least $0.13 \mathrm{mag}$ bluer than observed, and more so if there is internal reddening within the galaxy. Even with the zero-point uncertainties, we are confident that the objects are bluer than $(V-R) \sim 0.3$; with a reddening correction and/or if our zeropoints give colors which are too red (as suggested above), the objects might have an intrinsic $V-R$ closer to 0.0 .

Note that the magnitudes for the diffuse sources are upper limits because only a fraction of their light is measured with the small aperture. Inspection of the frames shows that the total brightness of these sources is not underestimated by more than a factor of 2 or so.

\subsection{Consistency of the Color Distribution with Continuum Sources}

A basic question is whether these objects are continuum sources or emission line sources. If they are the latter, one might expect to see $\mathrm{H} \alpha$ emission in one or the other of the narrowband filters. There are two $\mathrm{H} \alpha$ frames, at the appropriate wavelengths in the low and high velocity systems, although the high-velocity $\mathrm{H} \alpha$ filter is narrower than the low velocity one. The high velocity picture is severely underexposed and only the brightest object is seen, at a level consistent with its being a continuum source. On the low velocity
$\mathrm{H} \alpha$ frame, about a dozen objects are detected. Using the expected sensitivities of the filters, we computed what the instrumental colors in the filters should be for sources of spectral type A5 and F5. In Fig. 3 we compare these (solid lines) to the low-velocity $\mathrm{H} \alpha$ strengths relative to $\mathrm{F} 702 \mathrm{~W}$. The dotted line shows the expected color F664N-F702W for the case of a pure $\mathrm{H} \alpha$ source at $5200 \mathrm{~km} / \mathrm{s}$; the relative difference here just reflects the difference in transmission at the relevant wavelength between the two filters. Most of the fainter objects are not detected on the $\mathrm{H} \alpha$ frame, so only lower limits are shown.

Most of the brighter objects seem to have colors consistent with their being continuum sources of spectral type A or F with little or no excess $\mathrm{H} \alpha$ emission. The Shields-Filippenko object is clearly detected as an emission line source. The two other objects with apparent emission are found close to one another in a region of diffuse emission. Several other fainter objects may also have $\mathrm{H} \alpha$ excesses, but the errors of measurement are large. Although we are reasonably confident that most of the objects are continuum sources, this is an important point that should be confirmed with spectroscopic observations, which we hope to undertake soon.

\subsection{Spatial Distribution of Objects}

The spatial distribution of the objects appears to be roughly centered on the nucleus of the galaxy. They are centrally concentrated; almost all are found within 15 arcsec from the nucleus. They do not seem to be spatially associated with the high velocity system which lies to the northwest of the nucleus. We detect about 60 of them on the same chip with the galaxy, and only 10 more on all other chips combined. This concentration, combined with the fact that all other objects are blue, whereas most foreground stars should be red, strongly supports the conclusion that this population is physically associated with NGC 1275 . It is possible that the two fairly bright objects at $V-R=0.6$ and $V-R=0.5$ found on the adjoining chips are foreground stars, although they are rather faint for foreground stars of this color.

\subsection{Are These Objects Resolved?}

Because we lack an accurate PSF, we cannot determine whether the objects are resolved by subtracting a PSF and looking for residuals. As a substitute, we have chosen to take the difference of the aperture magnitudes at 2 and 4 pixel radii as an indication of the degree of resolution. Figure 4 plots the colors of the objects against this difference. In this plot, a single pixel source (e.g., a cosmic ray) would have a magnitude difference of zero; for a true point source, we expect the magnitude difference to be between 0.7 and 0.8 mag, as derived from the moderately bright star on PC5 and from the computed PSF models. Resolved sources will have larger magnitude differences.

Figure 4 demonstrates that the majority of objects have brightness distributions consistent with their being unresolved. This is difficult to be certain of, as the errors are rather large for most objects. By eye, the brightest objects appear to be point sources, and it is possible to see diffraction structure around them. The objects that are visibly diffuse reliably fall on the right-hand part of Fig. 4, indicating that the eye and quantitative estimates agree. We estimate the upper limit for the size of an unresolved source to be one pixel, which corresponds to $15 \mathrm{pc}$ in NGC 1275. This falls between the core radius and the tidal radius for typical Galactic globular clusters (Harris \& Racine 1979). 
TABLE 1. NGC 1275 photometry.

\begin{tabular}{|c|c|c|c|c|c|c|c|c|c|c|c|}
\hline ID $^{1}$ & $\Delta \mathrm{E}^{2}$ & $\Delta \mathrm{N}^{2}$ & $V$ & $\sigma_{V}$ & $V-R$ & $\sigma_{V-R}$ & $V 2-V 4^{3}$ & $\sigma_{V 2-V 4}$ & $\mathrm{H} \alpha-\mathrm{r}^{4}$ & $\sigma_{H \alpha-r}^{5}$ & Comments \\
\hline 1 & -1.5 & 3.1 & 18.2 & 0.02 & 0.33 & 0.03 & 0.72 & 0.27 & 3.1 & 0.1 & \\
\hline 3 & -2.2 & 2.3 & 19.2 & 0.03 & 0.32 & 0.06 & 0.72 & 0.36 & 3.0 & 0.2 & \\
\hline 2 & -1.3 & -1.9 & 19.3 & 0.08 & 0.27 & 0.16 & 0.70 & 0.59 & 3.0 & 0.2 & \\
\hline 501 & 26.0 & 12.2 & 19.5 & 0.01 & 0.60 & 0.02 & 0.69 & 0.25 & 3.2 & 0.1 & \\
\hline 701 & 5.2 & 30.5 & 19.6 & 0.02 & 0.54 & 0.03 & 0.72 & 0.30 & 3.1 & 0.2 & \\
\hline 4 & -3.4 & -0.7 & 19.8 & 0.06 & 0.41 & 0.11 & 0.73 & 0.53 & 3.2 & 0.2 & \\
\hline 5 & 3.4 & -4.1 & 19.9 & 0.04 & 0.24 & 0.07 & 0.70 & 0.40 & 3.1 & 0.2 & \\
\hline 6 & 0.1 & 4.6 & 20.0 & 0.06 & 0.33 & 0.10 & 0.74 & 0.51 & 3.2 & 0.2 & \\
\hline 502 & 22.2 & -3.0 & & $\ldots$ & & $\ldots$ & $\ldots$ & $\ldots$ & 2.5 & $*$ & \\
\hline 7 & -1.2 & 2.3 & 20.3 & 0.10 & 0.36 & 0.19 & 0.70 & 0.65 & 3.3 & 0.5 & \\
\hline 9 & -3.1 & -1.0 & 20.3 & 0.09 & 0.17 & 0.19 & 0.79 & 0.68 & 2.5 & 0.4 & \\
\hline 8 & 1.8 & -2.5 & 20.6 & 0.12 & 0.16 & 0.24 & 0.60 & 0.66 & 2.7 & 0.4 & \\
\hline 12 & -1.6 & 5.8 & 20.6 & 0.07 & 0.28 & 0.12 & 0.76 & 0.56 & 3.5 & $*$ & \\
\hline 11 & -1.4 & 1.2 & 20.6 & 0.37 & 0.60 & 0.67 & 0.36 & 0.92 & 3.5 & $*$ & \\
\hline 15 & -2.3 & 1.4 & 20.8 & 0.14 & 0.22 & 0.26 & 1.25 & 1.27 & 3.1 & $*$ & diffuse \\
\hline 503 & 17.1 & 9.1 & 20.9 & 0.05 & 0.45 & 0.08 & 1.06 & 0.62 & 1.4 & 0.1 & $S-F^{6}$ \\
\hline 19 & -0.7 & 7.2 & 20.9 & 0.07 & 0.17 & 0.13 & 0.79 & 0.59 & 2.7 & 0.9 & \\
\hline 13 & 2.0 & -3.5 & 20.9 & 0.11 & 0.28 & 0.21 & 0.78 & 0.74 & 3.2 & $*$ & \\
\hline 17 & -6.4 & 4.8 & 20.9 & 0.05 & 0.35 & 0.09 & 0.70 & 0.48 & $\ldots$ & $\ldots$ & \\
\hline 20 & 2.8 & 1.7 & 20.9 & 0.18 & 0.17 & 0.41 & 0.67 & 0.85 & $\ldots$ & $\ldots$ & \\
\hline 14 & -2.5 & 3.5 & 21.0 & 0.16 & 0.55 & 0.27 & 0.78 & 0.89 & 3.5 & $*$ & \\
\hline 24 & 4.6 & -2.7 & 21.1 & 0.11 & 0.20 & 0.21 & 0.70 & 0.70 & 2.9 & $*$ & \\
\hline 18 & -6.3 & 1.8 & 21.2 & 0.11 & 0.33 & 0.19 & 0.89 & 0.80 & 1.5 & 0.2 & \\
\hline 25 & -0.2 & -4.1 & 21.2 & 0.17 & 0.34 & 0.31 & 0.83 & 0.95 & 2.9 & $*$ & diffuse \\
\hline 21 & -1.9 & 2.8 & 21.3 & 0.22 & 0.22 & 0.96 & 0.53 & 0.82 & 2.6 & $*$ & \\
\hline 27 & -8.2 & 1.9 & 21.3 & 0.08 & 0.27 & 0.14 & 1.15 & 0.89 & 2.2 & 0.2 & diffuse \\
\hline 26 & 7.4 & -0.7 & 21.3 & 0.09 & 0.15 & 0.18 & 0.55 & 0.55 & 2.6 & * & \\
\hline 28 & 3.6 & -4.3 & 19.7 & 0.18 & $\ldots$ & $\ldots$ & 1.40 & 1.68 & $\ldots$ & $\ldots$ & \\
\hline 10 & 1.8 & -1.7 & 21.4 & 0.23 & 0.23 & 0.47 & 0.81 & 1.11 & $\ldots$ & $\ldots$ & \\
\hline 32 & -0.2 & 7.1 & 21.5 & 0.12 & 0.19 & 0.23 & 0.75 & 0.75 & 2.6 & $\cdot *$ & \\
\hline 29 & -5.5 & 1.4 & 21.5 & 0.10 & 0.36 & 0.21 & 1.27 & 1.11 & 1.6 & 0.3 & diffuse \\
\hline 33 & 3.8 & 0.6 & 21.5 & 0.17 & 0.30 & 0.29 & 0.79 & 0.93 & 2.6 & $*$ & \\
\hline 30 & -10.3 & -7.4 & 21.5 & 0.07 & 0.15 & 0.14 & 0.77 & 0.58 & 2.6 & $*$ & \\
\hline 23 & 2.8 & -0.9 & 21.5 & 0.31 & 0.23 & 0.58 & 0.85 & 1.33 & 2.4 & $*$ & \\
\hline 34 & 11.5 & 7.0 & 21.6 & 0.07 & 0.11 & 0.14 & 1.16 & 0.85 & 1.9 & 0.3 & diffuse \\
\hline 35 & 14.1 & -4.9 & 21.5 & 0.08 & 0.93 & 0.12 & 0.78 & 0.62 & 3.3 & 0.5 & \\
\hline 31 & 6.5 & -3.0 & 21.6 & 0.13 & 0.32 & 0.24 & 0.72 & 0.77 & 2.7 & * & \\
\hline 44 & 3.0 & 3.7 & 21.8 & 0.24 & 0.52 & 0.42 & 1.06 & 1.42 & 2.7 & $*$ & \\
\hline 37 & -3.6 & 7.3 & 21.8 & 0.11 & 0.44 & 0.20 & 0.58 & 0.62 & 2.7 & $*$ & \\
\hline 39 & -10.9 & 5.3 & 21.9 & 0.11 & -0.04 & 0.21 & 1.20 & 1.10 & 2.0 & $*$ & \\
\hline
\end{tabular}


TABLE 1. (continued)

\begin{tabular}{|c|c|c|c|c|c|c|c|c|c|c|c|}
\hline $\mathrm{ID}^{1}$ & $\Delta \mathrm{E}^{2}$ & $\Delta N^{2}$ & $V$ & $\sigma_{V}$ & $V-R$ & $\sigma_{V-R}$ & $V 2-V 4^{3}$ & $\sigma_{V 2-V 4}$ & $\mathrm{H} \alpha-\mathrm{r}^{4}$ & $\sigma_{H \alpha-r}$ & Comments \\
\hline 38 & -8.5 & 3.3 & 21.9 & 0.12 & -0.17 & 0.31 & 0.97 & 0.93 & 1.7 & $*$ & diffuse \\
\hline 36 & -3.4 & 1.0 & 22.0 & 0.41 & 0.85 & 0.66 & 0.47 & 1.07 & 2.8 & $*$ & \\
\hline 40 & -2.6 & 9.3 & 22.0 & 0.11 & 1.32 & 0.16 & 0.75 & 0.72 & 3.6 & 0.7 & \\
\hline 45 & 5.6 & -8.4 & 22.1 & 0.14 & 0.32 & 0.25 & 0.51 & 0.66 & $\ldots$ & $\ldots$ & \\
\hline 42 & -7.0 & 0.0 & 22.1 & 0.19 & 0.05 & 0.37 & 0.66 & 0.88 & $\ldots$ & $\ldots$ & \\
\hline 43 & -6.0 & 7.8 & 22.1 & 0.14 & 0.11 & 0.27 & 1.05 & 1.05 & 1.9 & $*$ & diffuse \\
\hline 46 & -9.2 & 2.9 & 22.2 & 0.16 & -0.57 & 0.47 & 1.03 & 1.14 & 0.4 & 0.8 & diffuse \\
\hline 48 & -6.3 & -22.3 & 22.1 & 0.12 & 0.72 & 0.19 & 0.92 & 0.89 & 2.4 & 0.7 & \\
\hline 50 & 5.4 & -6.1 & 22.2 & 0.20 & 0.20 & 0.39 & 0.54 & 0.80 & 1.9 & $*$ & diffuse \\
\hline 802 & 20.2 & 38.7 & 22.2 & 0.09 & 0.39 & 0.17 & 0.73 & 0.65 & 2.3 & $*$ & \\
\hline 16 & 1.4 & -1.7 & 20.7 & 0.41 & $\ldots$ & $\cdots$ & -1.05 & 0.26 & $\cdots$ & $*$ & \\
\hline 51 & 11.8 & 4.4 & 22.5 & 0.15 & -0.25 & 0.39 & 0.85 & 0.93 & 1.1 & $*$ & \\
\hline 49 & -8.2 & 7.9 & 22.4 & 0.18 & 0.53 & 0.38 & 0.87 & 1.03 & 2.2 & $*$ & diffuse \\
\hline 54 & -4.2 & 8.1 & 22.5 & 0.23 & 0.02 & 0.53 & 0.57 & 0.88 & 1.4 & $*$ & \\
\hline 53 & -8.1 & -6.2 & 22.5 & 0.19 & 0.41 & 0.32 & 0.26 & 0.60 & $\ldots$ & $\ldots$ & \\
\hline 56 & -4.9 & 7.5 & 22.6 & 0.22 & 0.04 & 0.48 & 0.37 & 0.72 & 1.2 & 0.6 & \\
\hline 52 & 11.1 & 11.5 & 22.6 & 0.20 & -0.08 & 0.55 & 0.90 & 1.11 & 1.2 & $*$ & diffuse \\
\hline 55 & 11.8 & 12.0 & 22.7 & 0.20 & 0.39 & 0.35 & 1.19 & 1.46 & 1.1 & 0.8 & diffuse \\
\hline 504 & 40.8 & 13.0 & 22.8 & 0.19 & 1.03 & 0.28 & 0.69 & 0.89 & 2.5 & $*$ & \\
\hline 703 & -1.0 & 26.3 & 22.9 & 0.25 & 0.18 & 0.56 & 1.26 & 1.74 & 1.3 & $*$ & \\
\hline 803 & 10.9 & 35.6 & 23.0 & 0.17 & 0.70 & 0.28 & 0.61 & 0.79 & $\ldots$ & $\ldots$ & \\
\hline 58 & 6.3 & -4.7 & 23.2 & 1.45 & 0.22 & 2.54 & -0.57 & 0.77 & 0.9 & * & \\
\hline 57 & 11.5 & 11.8 & 23.4 & 0.25 & 0.33 & 0.61 & 1.29 & 1.77 & 1.0 & $*$ & diffuse \\
\hline 41 & -3.2 & 2.7 & 23.6 & 0.56 & 0.81 & 1.11 & 1.39 & 2.91 & 0.7 & $*$ & diffuse \\
\hline
\end{tabular}

${ }^{1}$ ID numbering is roughly with brightness on chip 6 . Objects with ID numbers greater than 100 fall on other chips, with the chip given by the first digit.

${ }^{2}$ Position of object in arcseconds from the nuclear point source.

${ }^{3}$ Difference in instrumental magnitude between aperture of radii 2 and 4 pixels.

${ }^{4}$ Difference in instrumental magnitude between $\mathrm{H} \alpha$ filter $(\mathrm{F} 664 \mathrm{~N})$ and red filter (F702W).

${ }^{5}$ Asterisks denote an upper limit only.

${ }^{6}$ Shields-Filippenko object.

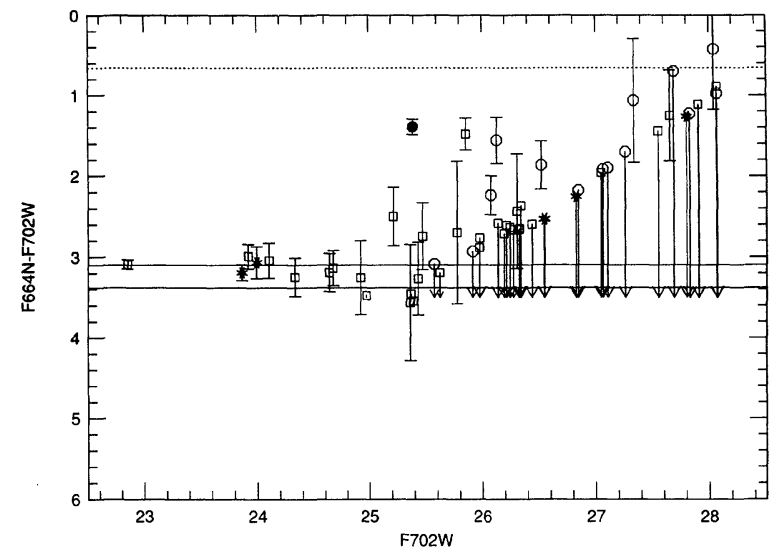

FIG. 3. Instrumental F664N vs F702W for the objects. The symbols are the same as for Fig. 2. The solid lines show the expected location of points for continuum sources of spectral type $A$ and $F$. The dotted line shows the expected location of a pure $\mathrm{H} \alpha$ source at the velocity of the NGC 1275 low-velocity system. Many of the fainter objects are not detectable on the F664N frame, so only lower limits are shown.

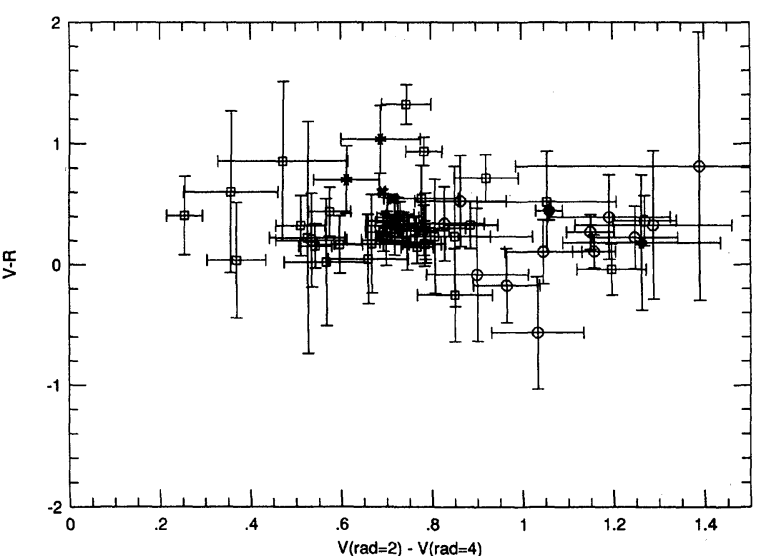

FIG. 4. $V-R$ vs the difference between the magnitudes of aperture radii of 2 and 4 pixels. Resolved source should fall on the right part of the plot. 
We find no strong trends in either brightness or color in going from point sources to diffuse sources. If anything, the diffuse sources may be slightly bluer than the compact ones, but given the errors, it is difficult to determine whether this is real.

\subsection{Galaxy Light and Color Distribution}

We turn next to an analysis of the underlying galaxy light. Figure 1 shows that the light distribution is patchy. The region where the high velocity gas is found lies to the northwest of the galaxy nucleus; some patchy structure is seen there. To the northeast, between the nucleus and the foreground star, there is clearly another fainter patch.

To enhance these features, we deconvolved a 1024 by 1024 section of the image using a model PSF. The same sections as are shown in Figs. 1(a) and 1(b) are shown in Figs. 5(a) and 5(b) [Plates 51 and 52]; the artifacts around the nucleus and foreground stars are caused by the inaccuracies of the PSF model. In the deconvolved image, the features mentioned above are readily apparent. In addition, there is a notable ripplelike structure in the galaxy which appears to extend from the foreground star to the southeast and then around the galaxy. This feature is visible on the raw frame with suitable contrast and can also be seen on ground-based frames [Lauer \& Postman (1991); also Burbidge \& Burbidge (1965), as noted by van den Bergh (1977)]. Another ripple can be seen due south of the nucleus and closer in.

We have also looked for color variations in the central region of NGC 1275. In general, regions that are fainter tend to be redder, which could result either from extinction or from an inhomogeneous population of blue stars. Inspection of the $V-R$ frame shows that the compact sources are unquestionably bluer than the underlying galaxy. We measure a color change of about 0.2 mag in $V-R$ from the outside of the PSF structure of the nucleus to the edge of the frame, about 20 arcsec from the nucleus. This is roughly consistent with the results of Romanishin (1987), who finds a difference of about $0.6 \mathrm{mag}$ in instrumental $B-I$ from the nucleus to a radius of 20 arcsec.

We cannot study the nuclear point source of NGC 1275 in detail because the broadband exposures saturate the few central pixels in the galaxy. We have measured a rough color by using the difference between the larger and smaller apertures and a correction based on PSF models, and also by looking at the profile of the wings. Transforming these measurements gives $V-R \sim 0.6-0.8$ with substantial uncertainties. The aperture photometry gives an estimate of $V \sim 15.1$ for the nuclear point source. The unresolved sources lie about 80 pixels from the nucleus and have an extent of one pixel at most, so their solid angles subtend only about $10^{-5}$ of the sky as seen from the nucleus. Since they are only a few magnitudes fainter than the nucleus, this rules out the possibility that they are simply reflecting light from the nucleus.

\section{INTERPRETATION}

\subsection{Star Clusters}

Since these objects are bright and have colors consistent with continuum sources, we believe that they are star clusters. The brightest objects are significantly brighter than any globular clusters seen in either our Galaxy or massive ellipticals such as M87; the most luminous globular in the Galaxy has $M_{V} \sim-10$ (Harris \& Racine 1979), while the brightest in M87 is a few magnitudes brighter (Strom et al. 1986).
Typical globulars have $M_{V} \sim-7$. Galactic globular clusters have intrinsic $V-R$ of 0.4-0.55 (Reed et al. 1988); their colors are correlated with metallicity, and we might expect that more metal rich globulars would be even redder. The NGC 1275 clusters have colors comparable with the blue clusters in the LMC but are significantly brighter; the brightest LMC cluster has $M_{V} \sim-10$ (van den Bergh 1981).

Bright clusters have been observed in other galaxies with luminosities comparable to those in NGC 1275, e.g., NGC 1569 (Arp \& Sandage 1990), NGC 1705 (Melnick et al. 1985), M82 (O’Connell \& Mangano 1978), NGC 3597 (Lutz 1991). Many of these have fairly large diameters and appear to be more similar to young associations than to compact primordial globular clusters, although a few are unresolved from ground-based observations.

It is particularly notable that most of the NGC 1275 objects, especially the brighter ones, appear to have approximately the same color. This suggests that they all formed within a fairly narrow time interval. We have determined ages and masses for the objects, assuming that each object is a star cluster whose members all formed at one time, using the stellar population synthesis models of Charlot and Bruzual (1991), which assume a Salpeter IMF and solar metallicities.

The age determination comes from the observed colors. Using an observed $V-R=0.30$ and a reddening $\mathrm{E}(V-R)=0.13$ gives an intrinsic $V-R=0.17$. In addition, there may be internal reddening within the galaxy; estimates of the total reddening have ranged from $\mathrm{E}(B-V)=0.1$ (Fabian et al. 1984) to $\mathrm{E}(B-V)=0.43$ (Kent \& Sargent 1979). The observed uniformity of colors, however, argues against the presence of a large amount of internal reddening. The color zeropoint is uncertain at a level of about a tenth of a magnitude, so a conservative estimate would be that the objects are bluer than $V-R=0.30$. Using the comparison with the galaxy color presented above and adopting large reddening corrections implies that they may be significantly bluer than this, possibly as blue as $V-R=0.0$

Figure 6 shows the evolution of the total $V$ and $V-R$ magnitudes of a single-burst population from Charlot \& Bruzual (1991; Charlot 1991); the Johnson $V-R$ has been transformed to the Cousins system using the transformation given by Bessell (1979). From this figure, we determine that the clusters are at most about 300 million years old, and perhaps as young as several million years. A similar comparison using the models of Struck-Marcell \& Tinsley (1978) gives maximum ages of about a billion years, although it is more difficult to make a comparison since these authors did not directly compute $V-R$. The general lack of strong $\mathrm{H} \alpha$ emission suggests that the objects are old enough that ionizing stars more massive than spectral type early $B$ have died, that high mass stars never formed, or that remaining gas has been ejected. The turnoff time for stars which might ionize $\mathrm{H}$ II regions is on the order of tens of millions of years.

In Fig. 7, we plot the observed luminosity distribution versus the current absolute magnitude on the bottom scale $\left(H_{0}=75 \mathrm{~km} \mathrm{~s}^{-1} \mathrm{Mpc}^{-1}\right)$. If the ages are about $300 \mathrm{mil}-$ lion years, the models predict that the $V$ magnitude will fade by about $4 \mathrm{mag}$ by the time these objects reach the age of present Milky Way globulars. If they are younger, they may fade by an additional few magnitudes. In Fig. 7, we show the 


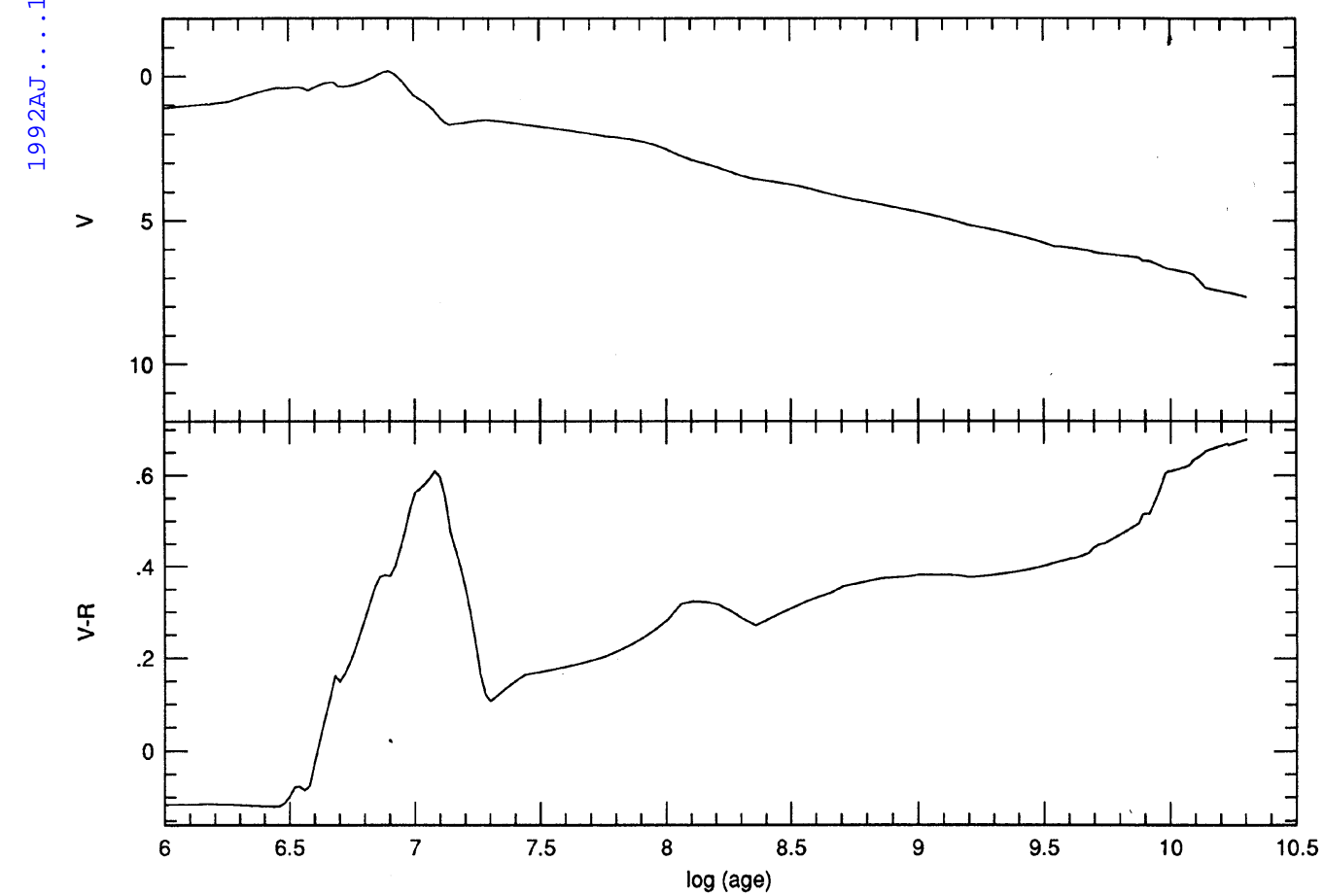

Fig. 6. Evolution of $V$ and $V-R$ for a single burst of star formation from the population synthesis models of Charlot \& Bruzual (1991).

"faded" magnitude scale along the top for a difference of 4 mag. The distribution of magnitude of Milky Way globulars is shown with the dotted line (Harris \& Racine 1979) for the upper scale. We find that it is plausible that the NGC 1275 objects will evolve to look very much like present day globulars, assuming that they are composed of normal stars with a "normal" IMF, and that they survive as distinct objects for the next 10-20 billion years. We have no measurements of metallicities for the NGC 1275 clusters but note that the broadband color and luminosity evolution is fairly insensitive to metallicity. The luminosity function suggests that either there are more clusters in NGC 1275 than in our Galaxy

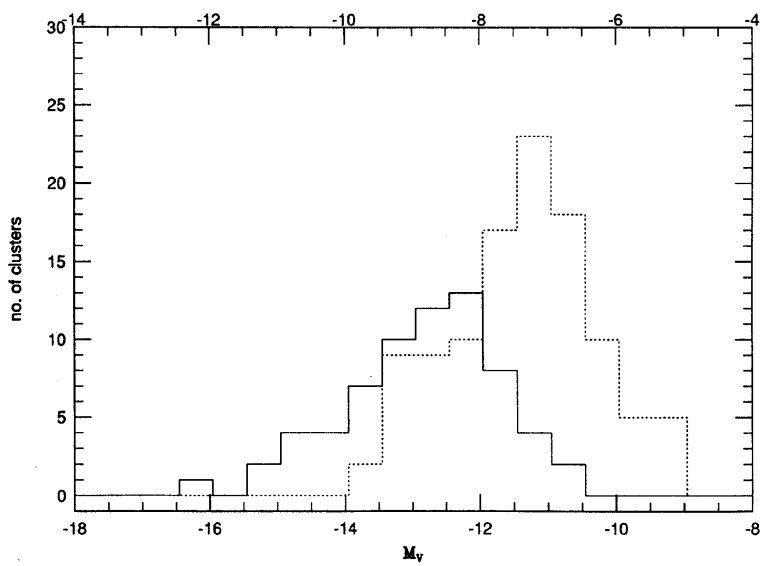

FIG. 7. The luminosity function of cluster objects. The dotted line shows the luminosity function for Milky Way globulars. The current absolute magnitude for the NGC 1275 objects is shown on the bottom scale. The top scale is relevant for the Milky Way clusters, and is the prediction for the NGC 1275 clusters if they were to fade by 4 mag. and we are incompletely detecting the fainter clusters, or that there is a comparable number but the brightest objects will fade by more than 4 mag. There are many fewer blue clusters associated with NGC 1275 than total clusters in M87, the central Virgo galaxy (which also has a cooling flow); however, there may be in addition a fainter population of older red globular clusters in NGC 1275 which have not been detected. An analogous blue population of clusters in M87 does not exist.

We have computed masses of the objects using the population synthesis models and find that the brightest object has a mass of between $2 \times 10^{6} \mathscr{M}_{\odot}$ and $1 \times 10^{8} \mathscr{M}_{\odot}$ for ages of $10^{7}$ and $3 \times 10^{8} \mathrm{yr}$. The next brightest objects are nearly a magnitude fainter and have masses that are a factor of $2 \frac{1}{2}$ lower. Most of the objects have inferred masses which are comparable to the masses of Milky Way globular clusters. Integrating up all of the brightnesses of the objects yields an approximate total mass of between $10^{7}$ and $6 \times 10^{8} \mathscr{M}_{\odot}$ in these clusters.

If these objects are to evolve to look like present day globulars, they must survive as distinct entities. Tidal disruption may occur, especially if the objects are in deeply plunging orbits. Such orbits may be expected given the inordinately low observed velocity dispersion of CO (around $110 \mathrm{~km} / \mathrm{s}$ ) seen in the center of NGC 1275 (Lazaref et al. 1989), assuming that the clusters formed out of this gas. The mass and compact size of the unresolved sources imply that they have densities comparable to Galactic globulars, and one might then expect the survival probability to be similar. On the other hand, some of the sources are visibly diffuse and probably have much lower densities and shorter tidal lifetimes.

We estimate the probability of tidal disruption by comparing the density of the clusters relative to the mean density of the galaxy interior to the clusters. We measure a rough galaxy surface brightness profile interior to the clusters and find that it is approximately inversely proportional to the radius, 
which implies an inverse square radial density distribution. Since the clusters are bluer than the galaxy light, we assume they have a lower mass-to-light ratio and adopt a value that is $1 \%$ of that of the underlying galaxy population. This is a lower limit obtained by assuming that the underlying galaxy has a mass-to-light ratio typical of an old globular cluster and the clusters have masses typical of globular clusters but are brighter by $5 \mathrm{mag}$; if the fading factor is less or if the clusters are more massive than Galactic globulars, then the mass-to-light ratio of the clusters will be larger relative to that of the galaxy. For the unresolved clusters (assuming they are marginally resolved), we then compute cluster densities which are 50-300 times the mean internal density, which implies that they are comfortably stable against tidal disruption at their current radii. If they are on deeply plunging orbits, they might disrupt since the galaxy might be very dense near the nucleus. A similar calculation for the resolved objects shows that they are likely to tidally disrupt where they are; indeed, these may be objects which have passed close to the nucleus and have already begun to disrupt.

Alternatively, all clusters could disrupt if their stars formed inefficiently out of denser clumps of material. In this case, the clusters could have large velocity dispersions and be unbound. However, as the internal disruption times are short, this would force all of the cluster ages to be young if one wanted to argue that all of the clusters were unbound. Also, this would require the presence of extremely massive clumps.

If the objects are in fact disrupting, one might expect to see clusters in various degrees of disintegration, with a tendency for the more diffuse objects to be older and redder. The measurements are uncertain, but if anything, the diffuse objects may be slightly bluer than the others, as seen in Figs. 2 and 4. Conversely, the presence of $\mathrm{H} \alpha$ emission in some of the diffuse objects might imply that these objects are younger than the unresolved clusters.

Strong hydrogen absorption lines have been detected in ground-based spectra of the body of NGC 1275 (Rubin et al. 1977; van den Bergh 1972), implying the presence of stars from mid-B to mid-A. IUE measurements (Nörgaard-Nielsen et al. 1990) and a large aperture measurement (Wirth et al. 1983) imply the presence of B stars. Given the locations of the spectral apertures as well as the brightnesses and locations of the clusters, it does not seem likely that the absorption seen from the ground can be attributed to the clusters, but rather that there is a more distributed population of young stars. An early type spectrum, however, is not seen at all locations in the galaxy. Because of the color zeropoint and age uncertainties for the clusters, it is not possible to determine whether the young stars in the galaxy are younger or older than those in the clusters. However, the known data are consistent with the two populations having formed at similar times.

We estimate the fraction of mass in the clusters to that in the distributed young background by assuming that the two populations have the same mass-to-light ratio and using the observational estimate of Wirth et al. that roughly $20 \%$ of the light in the inner 25 arcsec of NGC 1275 is in the form of young stars. We find that the mass in clusters is approximately $20 \%$ of the total mass in young stars, and greater if we make a correction for incompleteness of the cluster sample. This is significantly larger than the ratio of mass in $\mathrm{Ga}-$ lactic globulars to total spheroid mass, which is around $1 \%$. However, if all of the diffuse objects disrupt, the ratio in
NGC 1275 will decrease by something like a factor of 2 . To determine accurately whether the ratio of cluster to distributed mass will be higher in NGC 1275 than in other spheroidal systems requires better data for the faint end of the luminosity function of the clusters, a more accurate estimate of the total light in the distributed population, and a determination of whether the two populations are of comparable age. If the clusters are younger than the young background, they will fade more, and our estimate of the ratio of cluster mass to distributed mass will decrease.

\subsection{The Shields-Filippenko Object}

Shields \& Filippenko (1990; Shields et al. 1990) have recently detected a young star cluster in NGC 1275, and derive a mass of a few million solar masses. It appears that the Shields-Filippenko object is different from most of the other objects detected here. We observe the object on the edge of the PC5 chip; it is bright, but not among the brightest of our sources. It is also noticeably diffuse, and the red flux appears to be dominated by $\mathrm{H} \alpha$ emission, as demonstrated by the location of the object in Fig. 3. The observed broadband colors, however, are somewhat puzzling, since Fig. 2 shows that the object has $V-R$ comparable to the other objects, yet most of the $R$ flux comes from $\mathrm{H} \alpha$. Consequently, either this object has a very blue continuum, or else the $V$ filter is also contaminated by line emission (presumably [O III] and $\mathrm{H} \beta$ ). Either way its comparable $V-R$ is a coincidence. Two of the other objects which fall in the upper part of Fig. 3 with moderately small errors are close to one another in a region of diffuse emission located a few arcsec to the NW of the nucleus; these objects may represent subunits of another region like that discovered by Shields and Filippenko.

If the $\mathrm{H} \alpha$ emission is coming from an $\mathrm{H}$ II region, the presence of stars more massive than early B stars is implied so that ionizing photons be produced. This requires ages of less than several tens of millions of years for objects with emission. The fact that other objects do not have $\mathrm{H} \alpha$ emission implies that they are older, although it is possible that they are simply objects from which the gas has been ejected.

One might argue that the central nuclear source of NGC 1275 provides the ionizing photons to produce the $\mathrm{H} \alpha$ emission, but this seems unlikely given that the Shields-Filippenko object is among the furthest of the objects from the nucleus.

\subsection{Galaxy}

Of particular interest is the fact that the light distribution of the galaxy does not have regular structure. The galaxy has fainter regions which are also redder, which probably results from obscuration, either internal or foreground, or from variations in the stellar population. The fainter regions to the northwest of the nucleus are known to be obscured by the high-velocity system. The faintest and reddest regions, however, are found to the northeast and southwest. It is not clear whether these can be associated with obscuration from the high-velocity system or whether they indicate the presence of dust or a variation in stellar population within the main body of NGC 1275 .

The PC pictures also show the presence of ripplelike structures in the light distribution. The most prominent ripple is to the southeast of the nucleus. An additional fainter ripple can be seen somewhat closer to the nucleus toward the south when the picture is displayed with suitable contrast. This 
type of feature is reminiscent of those seen in galaxies that have undergone encounters with other galaxies (e.g., Schweizer 1986, and references therein), and we suggest that their presence in NGC 1275 implies that it has undergone an interaction in the recent past. Generally, these features are formed after an encounter and may persist in a galaxy for several dynamical times (Hernquist \& Quinn 1988,1989 ). Because shells form after encounters, it is unlikely that a current interaction with the high velocity system (which is still falling into NGC 1275) could cause the ripples. The discovery that NGC 1275 may have undergone a merger some $10^{7}-10^{8}$ yr ago adds yet another item to the list of known peculiarities in this system. However, it may provide a clue to unlocking much of its anomalous behavior.

\subsection{High-Velocity System}

The high-velocity system in NGC 1275 is found toward the north and northwest of the nucleus, extending all the way into the nucleus. It has been interpreted in various ways, ranging from suggestions.that it represents ejection from the nucleus, a superposition of a foreground spiral galaxy, or an interaction between a late-type galaxy and NGC 1275. Using long slit spectra, Boroson (1990) showed that the velocity structure across the high-velocity system is not as regular as had been previously claimed (Rubin et al. 1977) and consequently that there was likely to be an interaction taking place. Unger et al. (1990) reach a similar conclusion from Fabry-Perot observations of both velocity systems. van den Bergh (1977), however, questions whether it can be either a foreground galaxy or an interacting galaxy.

We had hoped to detect star light or $\mathbf{H}$ II regions from the high velocity system. A slight edge brightening of the dust lane due to starlight is possible but not certain.

\section{STAR FORMATION SCENARIOS}

We consider three scenarios to explain the recent star formation in NGC 1275, namely (1) it is being triggered by a current interaction with the high-velocity gas, $(2)$ it is occurring continuously in the cooling flow falling into the core of NGC 1275, and (3) it was triggered by a previous interaction of NGC 1275 with another system $10^{7}-10^{8}$ yr ago.

\subsection{Interaction with the High-Velocity System}

We know that there is an object in front of NGC 1275 falling toward the galaxy with a velocity of about $3000 \mathrm{~km} / \mathrm{s}$. A detailed study of the emission from the two velocity systems shows an anticorrelation between the brightest regions of the two systems, suggesting that the systems are interacting (Unger et al. 1990). Since interactions probably trigger star formation, we consider whether an ongoing collision might account for the young clusters and distributed stellar population in NGC 1275.

The main objection to this hypothesis is that the encounter velocity is quite high. At $3000 \mathrm{~km} / \mathrm{s}$, the systems will not merge, and the total encounter time will be on the order of only a few million years; since the high velocity system is a foreground object, only a fraction of this time could have already passed. This implies that all stars formed must be quite young and furthermore that they be located in the part of NGC 1275 that is being impacted by the high-velocity system. This does not appear to be the case. The cluster colors suggest larger ages, although very small ages might be possible if we push the zero points and reddenings to their limits. The lack of $\mathrm{H} \alpha$ emission in most of the clusters suggests that they are not very young. The presence of hydrogen absorption in the galaxy indicates that significant light is contributed by late B/early A stars, which is expected only after several tens of millions of years. As far as the spatial distribution is concerned, the clusters are roughly symmetrically distributed around the nucleus of NGC 1275 and show no concentration toward the high-velocity system. Finally, an interaction with the high-velocity system is also unable to explain the ripples seen in the galaxy light because the encounter velocity is too high and the timescale is too short.

Consequently, we find it unlikely that a current interaction can explain the observations. Unger et al. (1990) reached basically the same conclusion.

\subsection{Cooling Flow Star Formation}

$\mathrm{X}$-ray observations suggest that the mass accretion rate in cooling flows decreases toward the center of the flow (e.g., Thomas et al. 1987). Star formation has been considered a prime candidate for the eventual fate of the infalling material (McNamara \& O'Connell 1989). Optical observations of cooling flow galaxies often imply the existence of young stars, but the star formation rates are usually determined to be significantly smaller than the inferred cooling flow accretion rates if stars form with a normal IMF. This has led to the suggestion that star formation in cooling flows preferentially forms low-mass stars (e.g., Fabian et al. 1982).

Here we consider whether star formation in a cooling flow might account for the observed properties of NGC 1275 . We implicitly assume that such star formation would occur continuously rather than in bursts. We infer the total mass in the clusters using the population synthesis models. For a maximum age of 300 million years, we compute a total mass of about $6 \times 10^{8} \mathscr{M}_{\odot}$; if the maximum observed age were smaller, the inferred mass would also be smaller. In any case, the star formation rate seems to be only a few solar masses per year. Thus, even with a contribution from the distributed young stellar population included, it seems difficult to consign to star formation the entire mass that is inferred from the $\mathrm{x}$-ray observations, at least not if the IMF is anywhere near normal. On the other hand, if star formation is occurring much further out in the galaxy, or if the estimated accretion rates are lower than has been previously considered, it is possible that most or all of the cooling flow gas may form into stars. Recently cooler $\mathrm{x}$-ray gas has been detected in the central regions of cooling flow galaxies (White et al. 1991), which may lower the amount of material that must be transformed into stars in order to absorb the entire x-ray accretion rate.

The current observations do not support the hypothesis that low-mass stars are formed preferentially in cooling flows. The observed colors of the star clusters show that stars at least as massive as A stars exist in the clusters. The far UV spectrum of the underlying galaxy indicates the presence of mid-B stars. $\mathrm{H} \alpha$ emission in the Shields-Filippenko object, as well as in another region of diffuse emission, suggests that even more massive stars must exist in these locations.

The main objection to continuous star formation is the observed uniformity of colors of the clusters, with an apparent lack of clusters with ages greater than several hundred million years. If clusters formed continuously from the flow, one would expect to see a range of ages and a tendency for fainter clusters to be redder, since burst populations fade and redden as they age. In Fig. 2, an approximate fading vector is 
shown for single burst models. Our observations do not show a correlation between color and brightness, although higher accuracy measurements are desirable. One way to reconcile the observed color uniformity with continuous star formation is to suppose that all of the clusters tidally disrupt within several hundred million years, thus explaining the apparent lack of older clusters; this would require the observed clusters to be on deeply plunging orbits. In this case, we would expect the distributed background population to be older than the cluster population, which unfortunately will be difficult to detect observationally because the distributed population is contaminated by the (presumably older) population of stars from the original galaxy.

\subsection{Star Formation Triggered by a Previous Interaction}

Several observations suggest that an interaction of NGC 1275 with another object occurred some 10-100 million years ago and triggered star formation. This would explain the uniformity of colors observed among the star clusters and the observed structural peculiarities in the galaxy light distribution; it also could explain the regions of early type spectra seen in the underlying galaxy and the presence of ultraviolet continuum emission. The timescales inferred for star formation from any of the observations, as well as that for the production of structure, are comparable and on the order of $10^{7}-10^{8} \mathrm{yr}$.

There is ample evidence that star formation occurs in interacting or merging systems when there is gas present (e.g., Schweizer 1982, and references therein). In some cases, there may be evidence that clusters form in such systems (Lutz 1991; Ashman \& Zepf 1991; Schweizer 1982). In particular, the NGC 1275 clusters appear to be strikingly similar to those recently reported by Lutz (1991) in NGC 3597. NGC 3597 has 20-30 centrally concentrated clusters with $0.1 \lesssim V-R \lesssim 0.5$ with a median $M_{V} \sim-13$. Asymmetric structure in NGC 3597 strongly suggests that it is a merging system. Ground-based observations set an upper limit on the size of these clusters of about 100 pc. Besides the clusters, there is evidence for a distributed population of young stars in the center of NGC 3597.

The possibility of an age spread among the NGC 1275 clusters, as implied by the fact that only some of the objects have $\mathrm{H} \alpha$ emission, does not compromise the merger scenario, as it is possible that star formation might continue for some time after a merger has begun. The distribution of cluster ages might be bimodal, or even multimodal, with each peak corresponding to an intense period of star formation Recently formed clusters might thus be superimposed on an older substrate, including even some very old globulars that formed at the very beginning of the galaxy. More accurate photometry of the fainter clusters and an extension to lower luminosity is required to determine whether such older generations are present.

An ugly aspect of the merger scenario is that it adds another peculiarity to the list of known peculiarities of NGC 1275 without at the same time providing a unified hypothesis that explains them all collectively. The known abnormalities of NGC 1275 are already rare occurrences. In postulating a merger we are invoking yet another rare event, namely, the existence of a low-velocity, gas-rich colliding galaxy in a cluster where virtually all galaxies have high velocity and are gas poor. One aspect of this objection might be met if we imagined that the colliding galaxy might interact with the cooling flow gas and trigger star formation in it rather than in its own interstellar medium.

A merger origin for the star formation in NGC 1275 would have important implications for the hypothesis that elliptical galaxies formed by mergers. Many of these are discussed by Ashman \& Zepf (1991) and by Lutz (1991). In particular, the possibility that mergers trigger star formation in the central region of the merger would allow the central phase space density of the merger remnant (i.e., the elliptical galaxy) to increase over the densities of the merging galaxies. Also, if globular clusters are formed in mergers, one would no longer expect the specific frequency of globulars to remain invariant through merger events (Schweizer 1987; Burstein 1987), which has been raised as a major objection to the merger hypothesis (van den Bergh 1982).

This scenario might also be a model for how cluster/spheroidal populations form at high redshift. In NGC 1275 , we apparently see both cluster and diffuse populations forming with a large fraction of mass going into the clusters. With tidal disruption occurring, however, a cluster/spheroid mass ratio more similar to that of our own Galactic halo might gradually evolve.

\section{CONCLUSION}

Observations of NGC 1275 made with the Planetary Camera on the HST have revealed a population of bright blue pointlike sources. Narrowband $\mathrm{H} \alpha$ frames show that most of these are continuum sources, and we suggest that they are massive star clusters. If this is true, they are bluer than any globular clusters found in the Milky Way or M87 and more massive than the blue clusters of the LMC. Population synthesis models imply that they have ages of less than several hundred million years and corresponding masses of $10^{6}-10^{8} \mathscr{M}_{\odot}$. They are compact and most appear to be stable against tidal disruption. We suggest that these objects will evolve to look like "normal" globular clusters.

Zero-point uncertainties and large photometric errors prevent us from unambiguously determining a star formation history. The apparent uniformity of colors among the brightest objects, however, suggests that a burst of star formation may have occurred several tens or hundreds of millions of years ago. Structure is detected in the underlying galaxy light that is suggestive of a merger between NGC 1275 and a second galaxy some $10^{7}-10^{8}$ yr ago. Unless all the clusters disrupt, steady-state star formation in the $x$-ray cooling flow would imply a wider range in cluster age and color than is seen, although more accurate photometry is required to be sure of this. An interaction with the projected, high-velocity, infalling system definitely cannot explain the observations because it has not yet reached the center of NGC 1275, where the clusters are concentrated, and because this system also has a total interaction time that is far too short for either the observed cluster lifetimes or the dynamical lifetime of structure in the galaxy.

If a previous merger triggered star formation in NGC 1275 , we may be viewing an analog of the late stages of elliptical galaxy formation still in progress. The presence of recently formed protoglobulars caused by a merger would remove an important objection to the merger hypothesis for elliptical galaxy origins. The newly suspected merger event may have also provided fuel injection for the bright Seyfert nucleus seen in NGC 1275.

This research was conducted by the WFPC Investigation Definition Team, supported in part by NASA Grant No. NAS5-1661. 
Note added in proof. All magnitudes in this paper are too bright by a factor of 2; the colors are unchanged. This error was caused by an error in the calculation of the exposure times. The basic conclusions of the paper are unchanged. We thank Dennis Crabtree for drawing this to our attention, based on a comparison to his ground-based data.

Arp, H., \& Sandage, A. 1985, AJ, 90, 1163

Ashman, K. M., \& Zepf, S. E. 1991, STScI preprint No. 567

Bessell, M. S. 1979, PASP, 91, 589

Boroson, T. A. 1990, ApJ, 360, 465

Burbidge, E. M., \& Burbidge, G. R. 1965, ApJ, 142, 1351

Burstein, D. 1987, in Nearly Normal Galaxies, edited by S. M. Faber

(Springer, New York), p. 47

Burstein, D., \& Heiles, C. 1984, ApJS, 54, 33

Cardelli, J. A., Clayton, G. C., \& Mathis, J. S. 1989, ApJ, 345, 245

Charlot, S. 1991, private communication

Charlot, S., \& Bruzual, A. G. 1991, ApJ, 367, 126

Cowie, L. L., Hu, E. M., Jenkins, E. B., \& York, D. G. 1983, ApJ, 272, 29

De Young, D. S., Roberts, M. S., \& Saslaw, W. C. 1973, ApJ, 185, 809

Fabian, A. C., Hu, E. M., Cowie, L. L., \& Grindlay, J. 1981, ApJ, 248, 47

Fabian, A. C., Nulsen, P. J., \& Canizares, C. R. 1982, MNRAS, 201, 933

Fabian, A. C., Nulsen, P. J., \& Arnaud, K. A. 1984, MNRAS, 208, 179

Harris, H. C., Baum, W. A., Hunter, D. A., \& Kreidl, T. J. 1991, AJ, 101, 677

Harris, W. E., \& Racine, R. 1979, ARA\&A, 17, 241

Hernquist, L., \& Quinn, P. J. 1988, ApJ, 331, 682

Hernquist, L., \& Quinn, P. J. 1989, ApJ, 342, 1

Holtzman, J. A., Groth, E. J., Light, R. M., Faber, S. M., Hunter, D. A., O’Neil, E. J., Shaya, E. J., \& WFPC IDT 1991, ApJ, 369, L35

Hughes, D. H., \& Robson, E. I. 1991, MNRAS, 249, 560

Kent, S. M., \& Sargent, W. L. W. 1979, ApJ, 230, 667

Lauer, T. R. 1989, PASP, 101, 445

Lauer, T. R., \& Postman, M. 1991, from the WARPFIRE Project, private communication

Lazaref, B., Castets, A., Kim, D. W., \& Jura, M. 1989, ApJ, 336, L13

Lucy, L. B. 1974, AJ, 79, 745

Lutz, D. 1991, A\&A, 245, 31

Lynden-Bell, D., Faber, S. M., Burstein, D., Davies, R. L., Dressler, A., Terlevich, R. J., \& Wegner, G. 1988, ApJ, 326, 19

\section{REFERENCES}

Lynds, R. 1970, ApJ, 159, L151

McNamara, B. R., \& O’Connell, R. W. 1989, AJ, 98, 2018

Melnick, J., Moles, M., \& Terlevich, R. 1985, A\&A, 149, L24

Minkowski, R. 1957, in Radio Astronomy, IAU Symposium No. 4 , edited by H. C. van de Hulst (Cambridge University Press, Cambridge), Vol. 107

Nörgaard-Nielsen, H. U., Hansen, L., \& Jörgensen, H. E. 1990, A\&A, 240, 70

O’Connell, R. W., \& Mangano, J. V. 1978, ApJ, 221, 62

Reed, B. C., Hesser, J. E., \& Shawl, S. J. 1988, PASP, 100, 545

Romanishin, W. 1987, ApJ, 323, L113

Richardson, W. H. 1972, J. Op. Soc. Am. 62, 52

Rubin, V. C., Ford, W. K., Peterson, C. J., \& Oort, J. H. 1977, ApJ, 211, 693

Schweizer, F. 1982, Apj, 252, 455

Schweizer, F. 1986, Sci, 231, 227

Schweizer, F. 1987, in Nearly Normal Galaxies, edited by S. M. Faber (Springer, New York), p. 18

Shields, J. C., \& Filippenko, A. 1990, ApJ, 353, L7

Shields, J. C., Filippenko, A., \& Basri, G. 1990, AJ, 100, 1805

Strom, S. E., Forte, J. C., Harris, W. E., Strom, K. M., Wells, D. C., \& Smith, M. G. 1986, ApJ, 245, 416

Struck-Marcell, C., \& Tinsley, B. M. 1978, ApJ, 221, 562

Thomas, P. A., Fabian, A.C., \& Nulsen, P. E. J. 1987, MNRAS, 228, 973 van den Bergh, S. 1972, JRASC, 66, 237

van den Bergh, S. 1977, AN, 298, 285

van den Bergh, S. 1981, A\&AS, 46, 79

van den Bergh, S. 1982, PASP, 94, 495

Unger, S. W., Taylor, K., Pedlar, A., Ghataure, H. S., Penston, M. V., \& Robinson, A. 1990, MNRAS, 242, 33P

White, D. A., Fabian, A. C., Johnstone, R. M., Mushotzky, R. F., \& Arnaud, K. A. 1991, MNRAS, 252, 72

Wirth, A., Kenyon, S. J., \& Hunter, D. A. 1983, ApJ, 269, 102 
PLATE 49

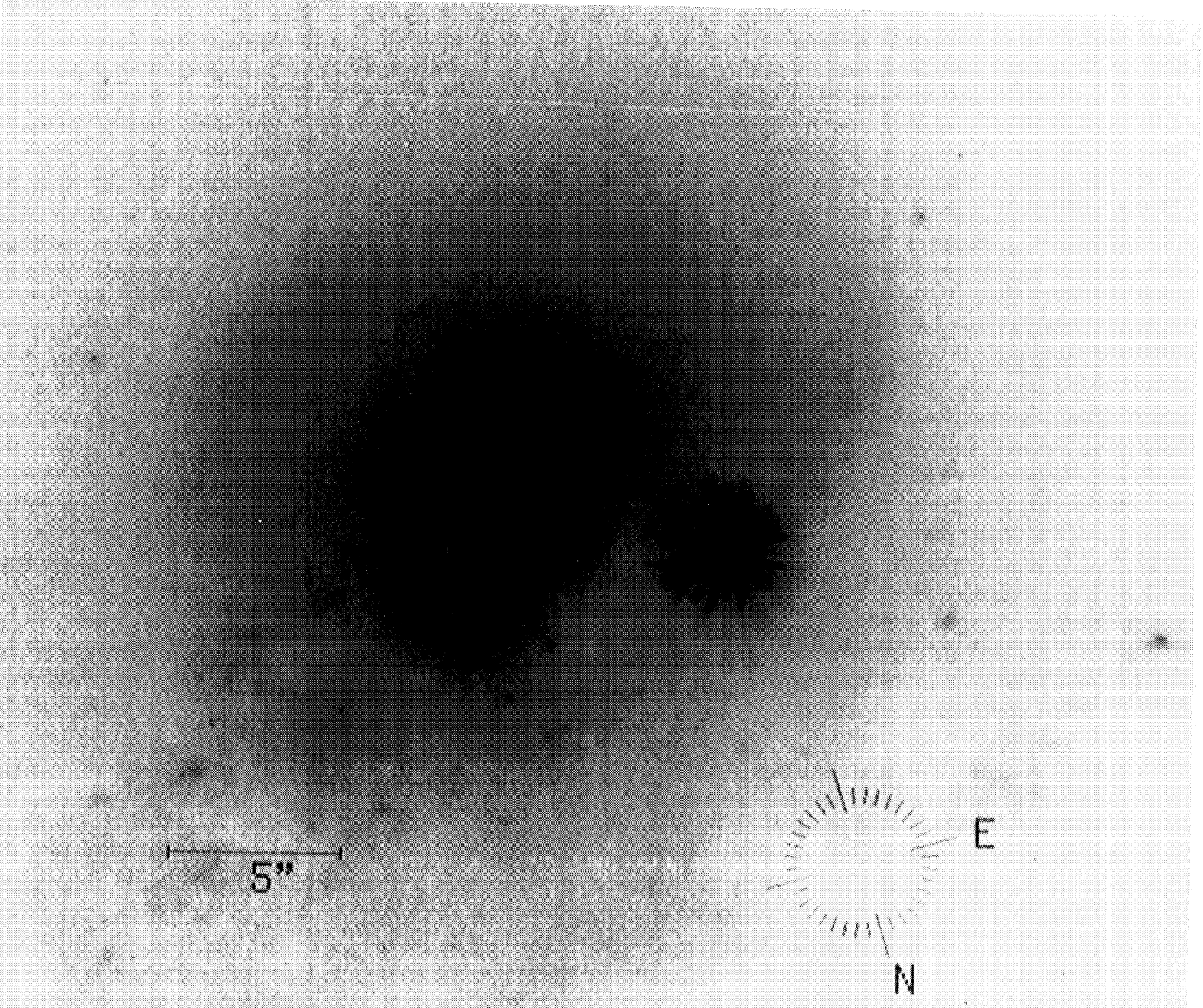

(a) FiG. 1. (a) A F555W image of NGC 1275 with logarithmic stretch. An $800 \times 800$ region, corresponding to $35 \times 35$ arcsec is shown. (b) A $17 \times 17$ arcsec
blowup of the central region of NGC 1275 .

Holtzman et al. (see page 692) 


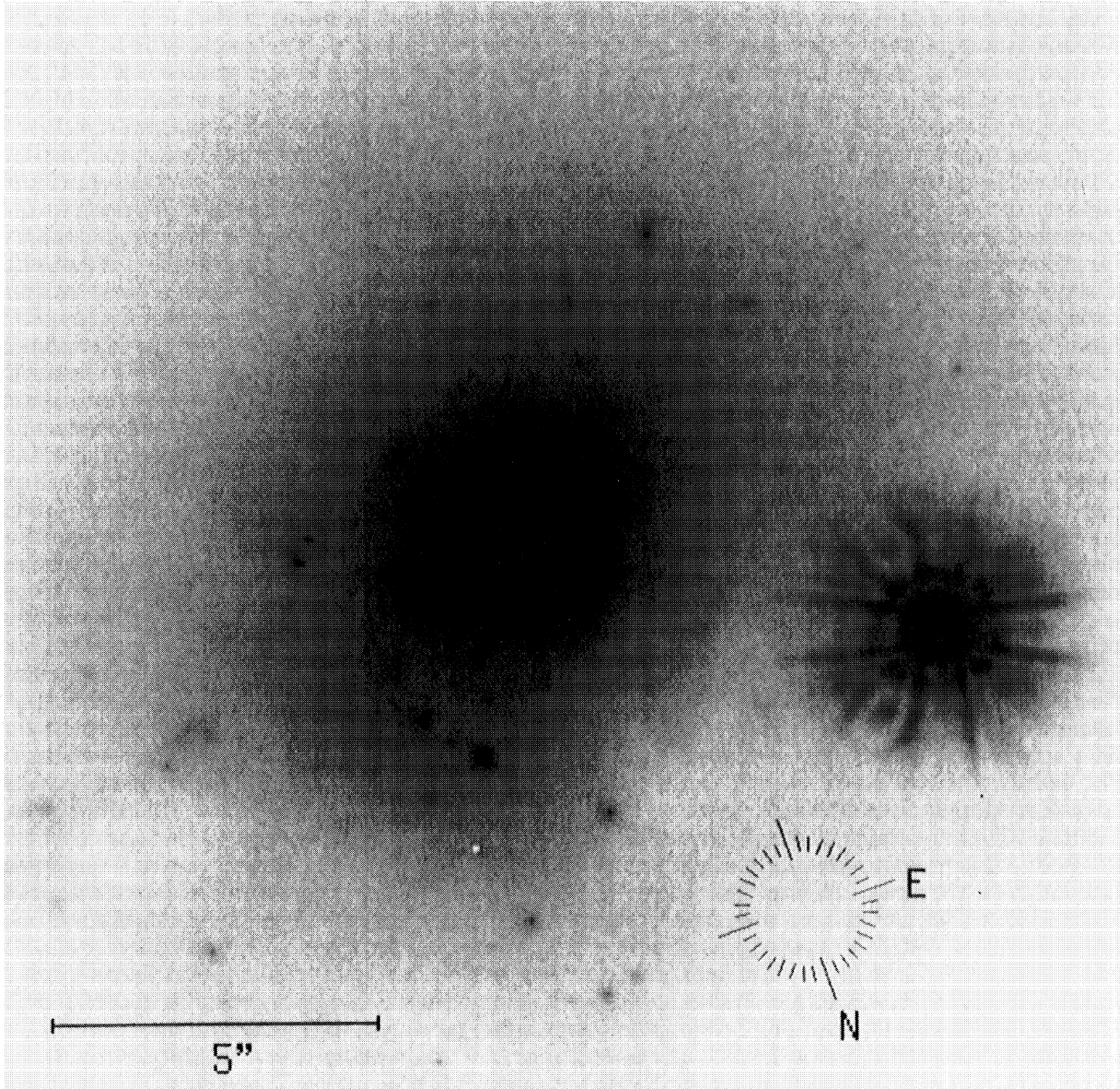

b)

FIG. 1. (continued)

Ioltzman et al. (see page 693) 


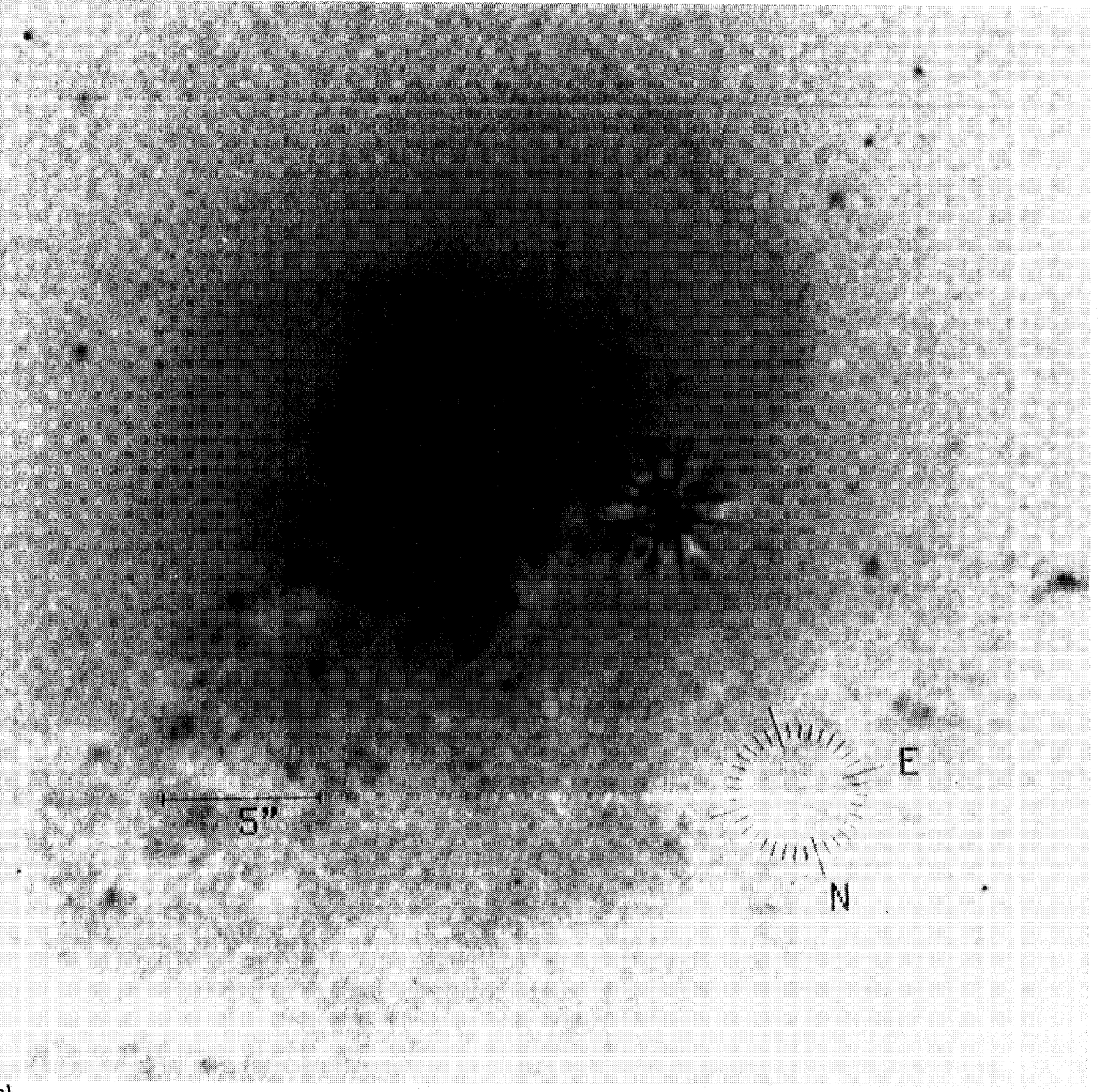

(a)

FIG. 5. (a) Deconvolved image of NGC 1275 shown in a log stretch. The deconvolution was performed using a model PSF with 20 iterations of the Richardson-Lucy (Richardson 1972; Lucy 1974) algorithm. The same region is shown as in Fig. 1 (a). (b) Blowup of the central region of NGC 1275 in the deconvolved image.

Holtzman et al. (see page 697) 


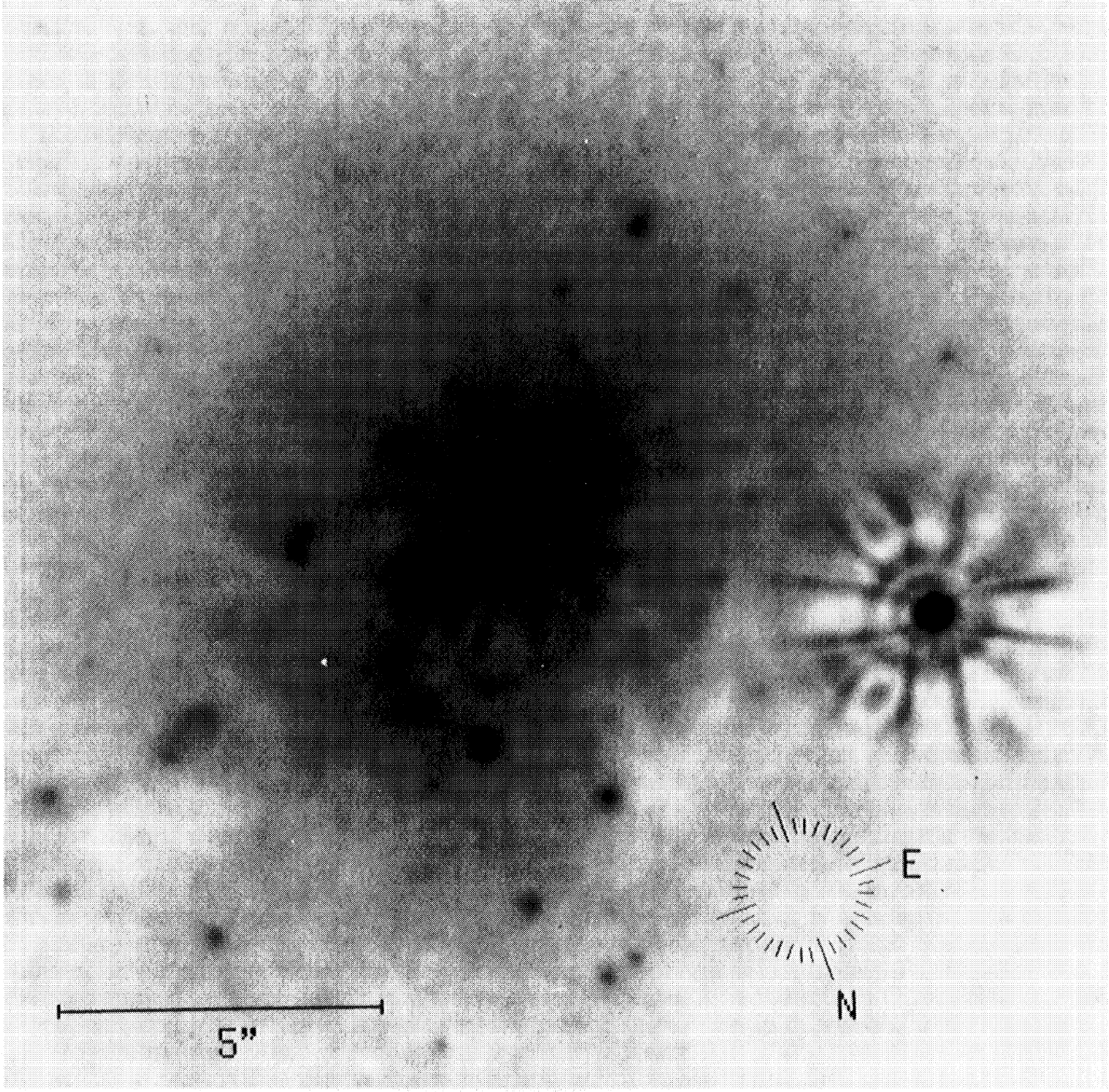

(b)

FIG. 5. (continued)

Holtzman et al. (see page 697) 